\title{
Autofocusing of Clinical Shoulder MR Images for Correction of Motion Artifacts
}

\author{
Armando Manduca, Kiaran P. McGee, E. Brian Welch, Joel P. Felmlee, and \\ Richard L. Ehman
}

Mayo Clinic and Foundation, Rochester MN 55905

\begin{abstract}
A post-processing "autofocusing" algorithm for the reduction of motion artifacts in MR images has been developed and tested on a large clinical data set of high resolution shoulder images. The algorithm uses only the raw (complex) data from the MR scanner, and requires no knowledge of the patient motion during the scan, deducing that from the raw data itself. It operates by searching over the space of possible patient motions and optimizing the image quality. Evaluation of this technique on the clinical data set (for which navigator echo based measured motions and corrected images were available) show that the algorithm can correct for the effects of global translation during the scan almost as well as the navigator echo approach and is more robust.
\end{abstract}

\section{Introduction}

The most serious remaining limitation in many current MRI examinations is data corruption by patient motion. Such motion causes phase errors in the received signal in k-space, which leads to ghosting, blurring and other artifacts in the image. A wide variety of techniques have been developed to minimize or correct for such motion, with perhaps the most successful being the use of navigator echoes [1]. However, corruption due to global patient motion does not actually lose information - if the motion is known, and the appropriate phase corrections applied, the image can be perfectly restored. It is therefore possible in principle to correct for motion given only the raw data from the MR scanner by simply trying different possible motion corrections and searching for the highest quality resulting image with a suitable evaluation function. Such approaches are used in the autofocusing of synthetic aperture radar images and in certain problems in seismic data processing.

Atkinson et al. [2] recently described such an algorithm, which uses entropy minimization as a focus criterion. We have developed a similar algorithm which we term "autocorrection", detailed below, and applied it to a clinical problem high-resolution shoulder imaging - in which the sharpness of the image is critical to the clinical evaluation of the supraspinatus tendon and rotator cuff. Navigator echoes have been shown to be effective on such images [3], and we used the data set from that study to directly compare autocorrection and navigator results. 


\section{Methods}

Autocorrection does not attempt to track patient motion (as do navigator-based methods of motion correction), nor does it seek information about the motion explicitly in the k-space data, as do some phase retrieval techniques. Rather, autocorrection algorithms perform motion correction by defining a measure or metric of image quality, and evaluating many combinations of possible patient motions, searching for a set which optimizes this quantity after the appropriate corrections are made to the image. It is implicitly assumed that the metric has an optimal value if the object is stationary, and that any motion during the imaging sequence will corrupt the image and degrade this value. In other words, it is assumed that it is impossible to make an image better (in terms of the metric) than the stationary case. It is also assumed that the better the value of the metric, the better the image.

In mathematical terms, autofocusing casts motion correction as an optimization problem, with the metric as the cost function, in a very high-dimensional space (as many dimensions as there are views in the acquired data). While it is probably impossible in practice to find the true global minimum of such a cost function, it appears to be possible to search the space of possible solutions in a way which yields very good improvements in image quality in a reasonable amount of time, as described below. It should be noted that similar approaches are used in the processing of synthetic aperture data [4] and in problems concerning the inversion of seismic data [5].

Atkinson et al. [2] recently showed the feasability of autofocusing for the reduction of MR motion artifacts, presenting an algorithm which used entropy minimization as the focus criterion. They considered both 2-D translation and rotation, and presented good results for simple test images. The algorithm we describe here is fundamentally similar to theirs, with improvements and differences as noted below. We have evaluated this autocorrection technique on high-resolution shoulder images, a clinical application in which maximum image sharpness is critical. Although this is a demanding test of the algorithm's ability to sharpen the image, it is also known that motion in such images is primarily translational along the superior-inferior direction [3]. This greatly simplifies the operation of the algorithm, since we need only consider one degree of freedom (motion along the phase-encoding direction).

\subsection{Optimization Algorithm}

The algorithm begins with the acquired k-space data (384 lines of 256 points each), and initially groups frequencies in blocks of 64 . A given block is temporarily "corrected for" a certain trial motion by applying the phase shifts that would correct for that motion, if the patient had in fact moved by that amount during the time corresponding to the acquisition of exactly those views. After these phase shifts, the data is transformed to image space and the metric is calculated. This metric is compared to the results for other trial motions, and, in this manner, the optimal motion correction for that block of frequencies can be 
determined. We use golden section optimization, and find the optimal motion to an accuracy of 0.1 pixel. This is done starting on one side of the center of $\mathrm{k}$ space and working outward, then moving to the other side and working outward (although alternating sides while moving outward seems to work equally well). When corrections are complete for the blocks of 64 , the process is begun again with blocks of 32 , and so on until individual lines are considered one at a time. This procedure allows one to gradually approximate the motion record more and more accurately as one moves to smaller block sizes. It is not critical to start at a block size of 64 (any large number is fine), and, perhaps surprisingly, it does not seem to be necessary to go all the way to considering each line individually. If one stops at blocks of 2 or even 4 lines, the correction is essentially complete one has already captured the major features of the motion record, and the finer details are not critical to the visual appearance.

The above paradigm of working with blocks of frequency space of gradually decreasing size has some similarities to the optimization strategy in [2]. Since they considered 2-D motions, however, they simply evaluated their metric on possible combinations of several discrete displacements in both $\mathrm{x}$ and $\mathrm{y}$, rather than using a more sophisticated optimization scheme in 1-D as we do here.

\subsection{Cost Function}

The most important improvement we have made is the choice of a better cost function: we use the entropy of the gradient of the image, rather than the entropy of the image itself. Entropy from information theory is defined in terms of the probability of a quantity assuming various values, and is minimized when most values have probability zero and a few values have high probability. In terms of an image, the entropy of the image itself (the metric proposed by [2]) is maximized when the image is dark in as large an area as possible, and its brightness concentrated in as few pixels as possible. It is known, however, that entropy is most sensitive to how close the small values are to zero, and is less sensitive to the behavior of the large values. Thus, entropy as an autofocusing metric depends critically on the dark areas of the image (especially minimizing ghosts in the background), and is not using information from bright areas very well. This was noted by [2], and we have confirmed that a significant amount of dark area is necessary to the success of image entropy based autofocusing on test images [6]. In the shoulder data set, initial experiments using image entropy as the metric failed to produce good motion correction - typically, ghosting in the background areas was reduced somewhat, but the image did not appear to sharpen sufficiently. It was also observed that changes in entropy values did not correlate well with observer judgments of how much an image was improving.

The metric used here (the entropy of the gradient) is minimized when the image consists of areas of uniform brightness, separated by sharp edges - since in such a case, the gradient is zero everywhere except at the edges, where it has high values. This is a fairly good model for what is expected in MR images of the body in ideal situations. Any blurring or ghosting will increase the entropy, since the gradient will be non-zero at more points and will take on smaller values 
at the actual edge locations. This metric thus takes full advantage of both bright and dark areas of the image. In a separate study [7], we compared the values of 24 separate metrics on the set of shoulder images described below both before and after navigator corrections, and correlated how well the change in the metrics predicted the improvement in image quality as judged by the observers. Gradient entropy had the smallest variance and gave the highest correlation with the observer ratings ( $R=0.53$, where $R=0.72$ was the inter-observer variability), and was much superior to image entropy $(R=0.33$, with high variance).

\subsection{Optimization on Selected Columns}

The algorithm in [2] requires a long computation time (many hours for a $256 \times 256$ image), due to the need for an inverse 2-D FFT for every new set of trial motions to evaluate the image in real space. In our case, this time is much reduced due to the single degree of freedom. However, still faster processing is possible by considering only selected columns of an image, since evaluating their quality requires only 1-D FFTs. We proceed from the $k$-space data by performing an inverse FFT in $\mathrm{x}$, transforming the data into hybrid space (spatial in $\mathrm{x}$, frequency in $y$ ). Since the only motion we are considering is along $y$, which is the phase-encoding direction, the phase correction terms do not depend on $\mathrm{x}$, and each column can be considered separately. We are therefore free to choose only columns of interest (in this case, those containing the humeral head), and need to inverse transform only those, greatly speeding up the calculations. We have to restrict the gradient used in the evaluation to consider only the y (phase encoding) direction, but it is in this direction that the ghosting occurs.

This approach also allows the calculations of corrections specific to a certain area of interest if the motion is not truly global. This is the case in the shoulder, since patient motion typically tends to be greater farther out towards the shoulder (the motion can be likened to a pump handle with the fulcrum along the central axis of the body) and the region of main clinical interest is relatively small. In synthetic test images, one can correct for induced motions with only a small number of columns [6]. In this clinical data set, we find a minimum of 32 to 64 columns seem to be necessary for stable motion correction, probably due to signal to noise considerations. We will report on this in more detail in the future.

\section{Evaluation}

The navigator study data set described in [3] comprises one hundred and forty four (144) high-resolution shoulder exams acquired under routine clinical conditions using an interleaved navigator pulse sequence. These were oblique coronal acquisitions with a double spin-echo sequence to provide proton-density and T2-weighted images. Further details of the acquisitions are provided in [3]. Raw $\mathrm{k}$-space data representing a slice bisecting the rotator cuff was selected from each 
of the 144 exams for autocorrection. The original, autocorrected, and navigatorcorrected images for the selected slices were printed onto film and evaluated by 4 observers experienced in reviewing shoulder images. For each case, they were asked to rank each image on the same scale of one through five $(1=$ non- diagnostic image quality, $2=$ severe motion effect, $3=$ moderate motion effect, $4=$ mild motion effect, and $5=$ no observable motion corruption) as used in [3]. The general results were that both correction techniques (autocorrection and navigator echoes) significantly improve the images, and while the navigator corrections are slightly better overall, the autocorrection corrections are nearly as effective.

Figure 1 shows the percentage of images which received each rating (from 1 to 5) for the original images and the two correction techniques. Both correction techniques significantly reduce the percentage of images with low ratings, increase the percentages with high ratings, and skew the distribution of ratings strongly towards the "excellent quality" side as compared to the original distribution. For the original images, only $23 \%$ were judged to be free of observable motion corruption. Autocorrection and navigator echoes both increased this value to $39 \%$. Conversely, $48 \%$ of the original images showed moderate motion corruption or worse (ratings of 1-3). This number was reduced to $27 \%$ after navigator correction and $28 \%$ after autocorrection.

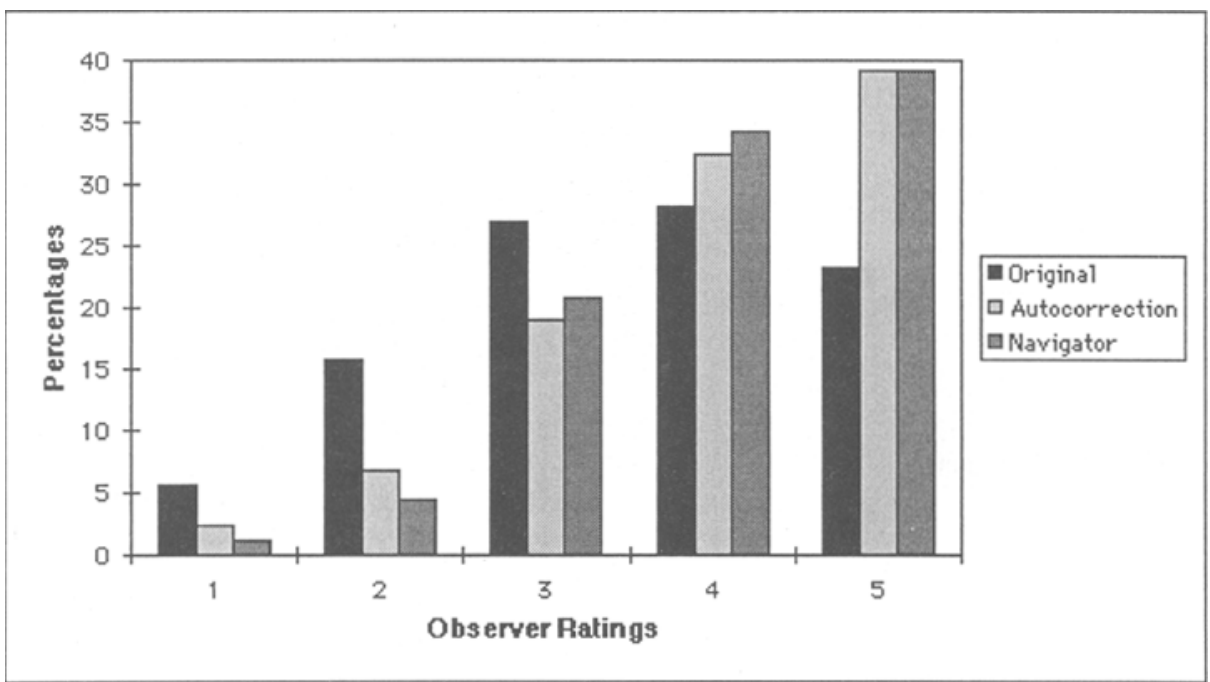

Fig. 1. Absolute observer ratings for images. Percentages of images receiving ratings of 1 (non-diagnostic image quality) through 5 (no observable motion corruption) are plotted for original images (black), autocorrected images (light gray), and navigator corrected images (dark gray). Both correction techniques significantly reduce the percentage of images with low ratings, increase the percentages with high ratings, and skew the distribution of ratings strongly towards the "excellent" side as compared to the original distribution. 
Table 1 shows the changes in observer rating when the correction techniques were applied to the original images. Navigators were judged to have improved the image 307 times vs. 279 for the autocorrection (out of 576 total ratings). Autocorrection, however, was more robust: the navigator correction was judged to have degraded an image 6 times, while autocorrection was never judged to have degraded an image. Table 2 shows the average improvement in the observer ratings for an image for each technique. Images with an original rating of 5 were not considered in the averages, since they could not possibly be improved and were never degraded by either technique. The overall average improvement with autocorrection (0.71) is more than $90 \%$ of the average improvement achieved with navigator echoes (0.78). Figure 2 shows two examples of successful corrections for each technique.

Table 1. Changes in Observer Ratings for Correction Techniques

\begin{tabular}{lll}
\hline Change in score & Autocorrection & Navigator \\
\hline-2 & 0 & 0 \\
-1 & 0 & 6 \\
0 & 297 & 263 \\
+1 & 247 & 262 \\
+2 & 32 & 45 \\
\hline
\end{tabular}

Table 2. Average Improvement in Observer Ratings

\begin{tabular}{lll}
\hline Observer & Autocorrection & Navigator \\
\hline Observer 1 & 0.69 & 0.70 \\
Observer 2 & 0.58 & 0.76 \\
Observer 3 & 0.89 & 0.92 \\
Observer 4 & 0.66 & 0.75 \\
\hline Average & 0.71 & 0.78 \\
\hline
\end{tabular}

\section{Discussion}

It is clear that autocorrection performs well in this application to high resolution shoulder MRI, perhaps remarkably so when one considers that it has no 
knowledge of the actual patient motion. The effectiveness of the algorithm presented here is perhaps $90 \%$ that of navigator echo based correction, and (unlike navigators) autocorrection never degraded an image. It is possible that this performance will improve with future refinements of the algorithm.
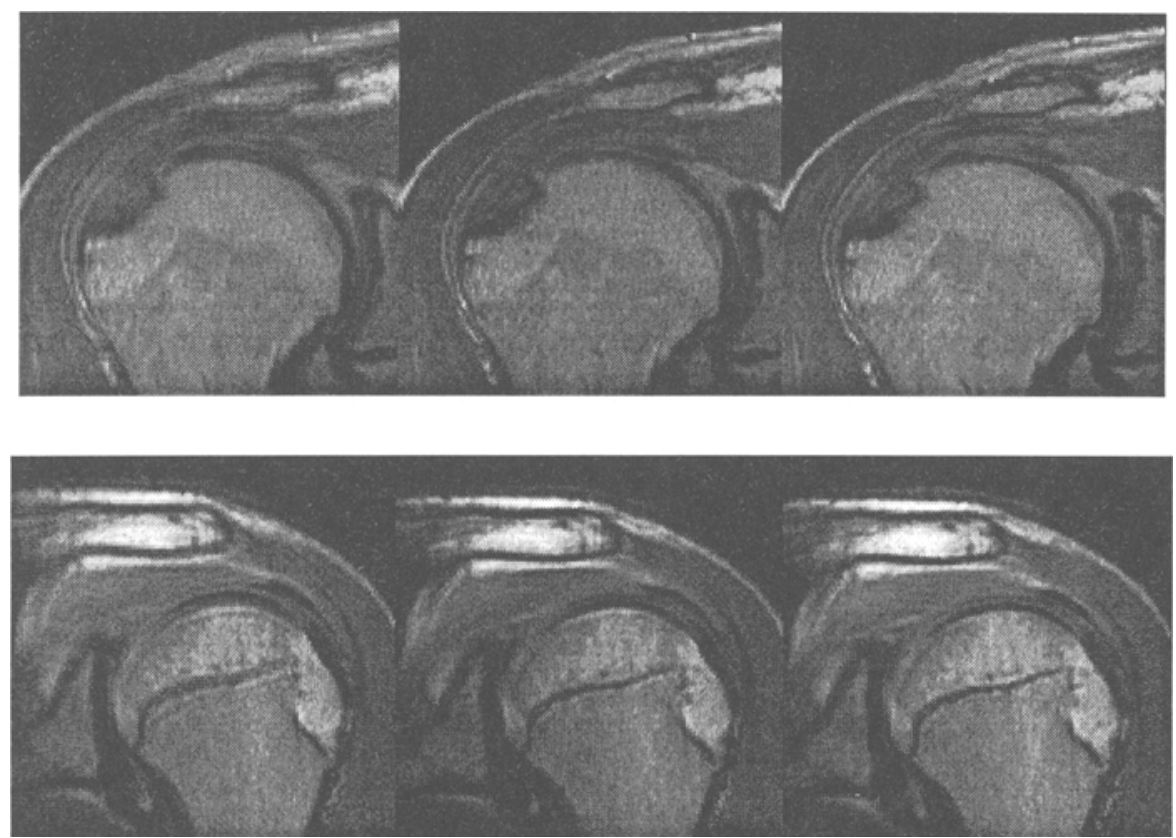

Fig. 2. Two sample images showing the original (left), autocorrected (middle), and navigator corrected versions (right). In these cases both techniques were judged to improve the image by +2 points.

We did not attempt to measure the effect of autocorrection on diagnostic accuracy, due to the difficulty of obtaining an independent test to establish the diagnosis. However, the improvements in image quality are readily apparent, and they are obtained without additional acquisition time, special pulse sequences, special hardware, or any sort of patient preparation. The autocorrection algorithm uses only the raw (complex) data from the scanner, and can be applied retrospectively to any data set from any scanner for which that information was saved, even data that is years old.

The computation times of the algorithm as used here are long, typically 15 minutes per slice on a Dec Alpha. We believe these times can be considerably shortened without affecting the quality of the result. Current experiments indicate that a time of 2-3 minutes per slice may suffice to give the level of performance achieved here. Future improvements in computing hardware and in the algorithm should further reduce computation time. 
The autocorrection approach can be readily extended to more complicated motions. Atkinson et al. (21) demonstrated corrections for 2-D translations and rotation, although their work was on a few test images without pathology. We are presently extending the algorithm to include 3-D translations and rotations, and will report such results in the future. We are also researching similar approaches to correct other, non-motion types of artifacts, such as timing errors in EPI images or saturation effects in gated cardiac imaging.

\section{Conclusion}

Autocorrection has been shown to be a practical technique for the reduction of motion artifacts in a demanding clinical application. The algorithm presented here uses only the raw (complex) data from the scanner, requires 15 minutes of processing time per slice, and significantly reduces motion artifacts. It performs nearly as well as the navigator echo technique, which is remarkable considering that the navigator is explicitly tracking patient motion while autocorrection uses no motion information. The algorithm never degraded an image, while the navigator did so $1 \%$ of the time. The algorithm is flexible and should be easily extensible to other types of motion in other applications and, quite possibly, to other types of artifacts in MR images.

\section{References}

1. Ehman RL, Felmlee JP. Adaptive technique for high-definition MR imaging of moving structures. Radiology 1989; 173:225-263.

2. Atkinson D, Hill DL, Stoyle PN, Summers PE, Keevil SF. Automatic correection of motion artifacts in magnetic resonance images using an entropy focus criterion. IEEE Trans Med Img 1997; 16(6):903-910.

3. McGee KP, Grimm RC, Felmlee JP, Rydberg JR, Riederer SJ, Ehman RL. The shoulder: Adaptive motion correction of MR images. Radiology 1997; 205:341-354.

4. Bocker RP, Jones SA. ISAR motion compensation using the burst derivative measure as a focal quality indicator. Int J Imag Syst Technol 1992; 4:285-297.

5. Stoffa PL, Sen MK. Nonlinear multiparameter optimization using genetic algorithms: inversion of plane-wave seismograms. Geophysics 1991; 56:1794-1810.

6. Welch EB, Manduca A, Ehman RL. Fast autofocusing of motion-corrupted MR images using one-dimensional Fourier transforms. Proc SPIE Med Img 1998; 3338: 391-397.

7. McGee KP, Manduca A, Felmlee JP, Grimm RC, Riederer SJ, Ehman RL. Autocorrection of high resolution shoulder MR images: analysis of image metrics. Proc ISMRM 1998. 